\title{
Anti-damping spin transfer torque through epitaxial nickel oxide
}

\section{$\operatorname{AUTHOR}(S)$ :}

Moriyama, Takahiro; Takei, So; Nagata, Masaki; Yoshimura, Yoko; Matsuzaki, Noriko; Terashima, Takahito; Tserkovnyak, Yaroslav; Ono, Teruo

\section{CITATION:}

Moriyama, Takahiro ...[et al]. Anti-damping spin transfer torque through epitaxial nickel oxide. Applied Physics Letters 2015, 106(16): 162406.

\section{ISSUE DATE:}

2015-04-22

URL:

http://hdl.handle.net/2433/198879

\section{RIGHT:}

(C) 2015 American Institute of Physics. This article may be downloaded for personal use only. Any other use requires prior permission of the author and the American Institute of Physics. 


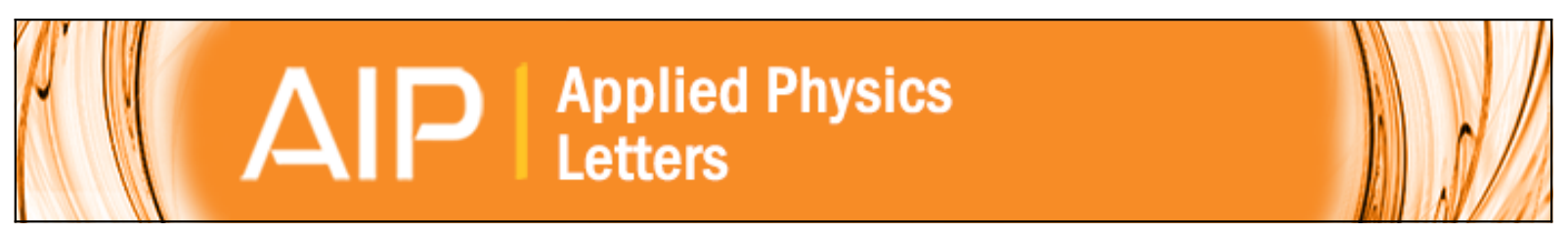

\section{Anti-damping spin transfer torque through epitaxial nickel oxide}

Takahiro Moriyama, So Takei, Masaki Nagata, Yoko Yoshimura, Noriko Matsuzaki, Takahito Terashima,

Yaroslav Tserkovnyak, and Teruo Ono

Citation: Applied Physics Letters 106, 162406 (2015); doi: 10.1063/1.4918990

View online: http://dx.doi.org/10.1063/1.4918990

View Table of Contents: http://scitation.aip.org/content/aip/journal/apl/106/16?ver=pdfcov

Published by the AIP Publishing

\section{Articles you may be interested in}

Localized excitation of magnetostatic surface spin waves in yttrium iron garnet by shorted coaxial probe detected via spin pumping and rectification effect

J. Appl. Phys. 117, 153903 (2015); 10.1063/1.4918668

Quantification of the spin-Hall anti-damping torque with a resonance spectrometer

Appl. Phys. Lett. 106, 022406 (2015); 10.1063/1.4906062

Determination of intrinsic spin Hall angle in $\mathrm{Pt}$

Appl. Phys. Lett. 105, 152412 (2014); 10.1063/1.4898593

Thickness dependence of spin torque ferromagnetic resonance in Co75Fe25/Pt bilayer films Appl. Phys. Lett. 104, 072405 (2014); 10.1063/1.4865425

Spin transport parameters in metallic multilayers determined by ferromagnetic resonance measurements of spinpumping

J. Appl. Phys. 113, 153906 (2013); 10.1063/1.4801799

Frustrated by

old technology?

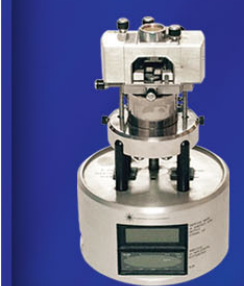

Is your AFM dead

and can't be repaired?

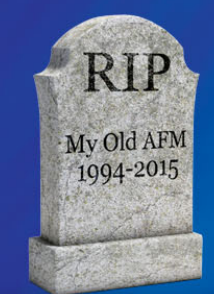

Sick of bad customer support?

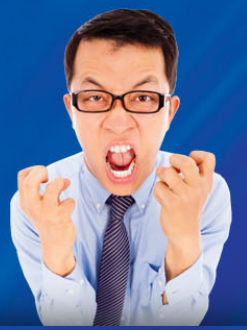

It is time to upgrade your AFM

Minimum $\$ 20,000$ trade-in discount for purchases before August 31st

Asylum Research is today's technology leader in AFM 


\title{
Anti-damping spin transfer torque through epitaxial nickel oxide
}

\author{
Takahiro Moriyama, ${ }^{1}$ So Takei, ${ }^{2}$ Masaki Nagata, ${ }^{1}$ Yoko Yoshimura, ${ }^{1}$ Noriko Matsuzaki, ${ }^{1}$ \\ Takahito Terashima, ${ }^{3}$ Yaroslav Tserkovnyak, ${ }^{2}$ and Teruo Ono ${ }^{1}$ \\ ${ }_{1}^{1}$ Institute for Chemical Research, Kyoto University, Uji, Kyoto 611-0011, Japan \\ ${ }^{2}$ Department of Physics and Astronomy, University of California, Los Angeles, California 90095, USA \\ ${ }^{3}$ Research Center for Low Temperature and Materials Sciences, Kyoto University, Kyoto 606-8501, Japan
}

(Received 9 February 2015; accepted 12 April 2015; published online 22 April 2015)

\begin{abstract}
We prepare the high quality epitaxial $\mathrm{MgO}(001)[100] / \mathrm{Pt}(001)[100] / \mathrm{NiO}(001)[100] / \mathrm{FeNi} / \mathrm{SiO}_{2}$ films to investigate the spin transport in the $\mathrm{NiO}$ antiferromagnetic insulator. The ferromagnetic resonance measurements of the FeNi under a spin current injection from the Pt by the spin Hall effect revealed the change of the ferromagnetic resonance linewidth depending on the amount of the spin current injection. The results can be interpreted that there is an angular momentum transfer through the NiO. A high efficient angular momentum transfer we observed in the epitaxial $\mathrm{NiO}$ can be attributed to the well-defined orientation of the antiferromagnetic moments and the spin quantization axis of the injected spin current. (C 2015 AIP Publishing LLC.
\end{abstract}

[http://dx.doi.org/10.1063/1.4918990]

Since the theoretical predictions ${ }^{1,2}$ followed by experimental demonstrations, ${ }^{3,4}$ spin transfer torque (STT) has been an efficient and promising technique to control magnetizations of ferromagnetic materials in modern spintronics devices. This novel technique is based on an interaction between electron spin and local magnetic moments. Namely, the angular momentum of the electron spin is transferred to and exerts a torque on the magnetization. The same interaction should be conserved in antiferromagnets (AFMs), in which there are microscopic local magnetic moments that compensate each other to exhibit no net magnetization. ${ }^{5-8}$ As AFMs have been abandoned as an active material in spintronics in spite of their potential applications in the $\mathrm{THz}$ regime, ${ }^{9}$ it is of great interest to investigate the STT in AFMs.

Despite numerous theoretical works on STT in AFMs, there are only a few experiments indicating the possibilities of interactions between the electron spin and the AFM moments. ${ }^{10-13}$ The most recent investigations of spin transport in AFM materials have raised an interesting question, whether AFMs can be transparent to the angular momentum flow. ${ }^{12,13}$ Wang et al. ${ }^{13}$ performed spin pumping measurements on $\mathrm{Y}_{3} \mathrm{Fe}_{5} \mathrm{O}_{12} / \mathrm{NiO} / \mathrm{Pt}$, and a spin voltage signal was detected by the inverse spin Hall effect in Pt. Spin transfer in a metallic AFM system with a $\mathrm{Pt} / \mathrm{IrMn} / \mathrm{FeCoB}$ heterostructure has also been investigated by our group by means of the spin-torque ferromagnetic resonance (ST-FMR). ${ }^{12}$ In the ST-FMR measurements, it was found, by investigating the change in linewidth, that the spin current injected from the $\mathrm{Pt}$ can give a spin torque on the $\mathrm{FeCoB}$ via the IrMn. It is quite remarkable that indications of spin transfer through AFMs have been confirmed by these two different techniques.

Those observations mostly draw only macroscopic interpretation of the interaction between the AFM moments and the spin current, because the Neel vectors are most likely randomly oriented with respect to the spin current due to polycrystallinity of the AFMs. In order to understand the microscopics of the interaction between AFM moments and the spin current, it is more desirable to investigate a clean and ordered structure that possesses well-defined AFM moments.

In this letter, we prepared $\mathrm{MgO}(001)$ substrate $/ \mathrm{Pt} / \mathrm{NiO} /$ $\mathrm{FeNi} / \mathrm{SiO}_{2}$ multilayers, in which the films are epitaxially grown until the NiO layer, and performed a ST-FMR measurement to quantify the anti-damping spin torque transported between the $\mathrm{Pt}$ and the $\mathrm{FeNi}$ through the $\mathrm{NiO}$ layer. In order to investigate the spin torque in $\mathrm{NiO}$, we created and injected a pure spin current by the spin Hall effect in Pt. The schematic layer structure of the injection scheme is shown in Fig. 1(b). The electron flowing in the Pt layer experiences a spin dependent scattering due to spin-orbit interaction, resulting in the opposite flow of spin polarized electrons orthogonal to the electron flow. ${ }^{14}$ This spin current is a so-called pure spin current, which does not involve charge current flow. This spin current induces a spin accumulation at the $\mathrm{Pt} / \mathrm{NiO}$ interface and exerts a spin torque on the $\mathrm{NiO}$ magnetic moments in a similar manner to the $\mathrm{Pt} /$ ferromagnetic insulator case. ${ }^{15}$ We investigate the magnetic damping

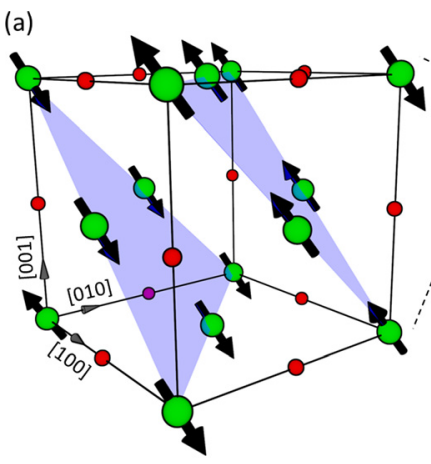

(b)

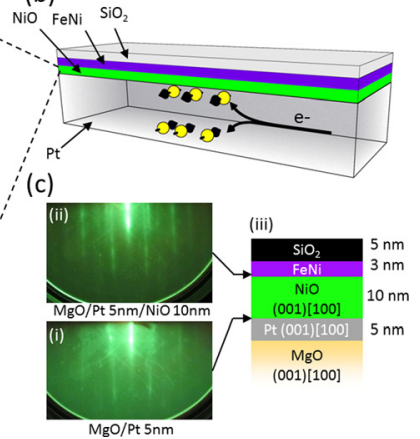

FIG. 1. (a) Crystal lattice of NiO. Green and red spheres indicate the Ni and $\mathrm{O}$ atoms, respectively. Magnetic moments reside at the $\mathrm{Ni}$ atoms. (b) Schematic illustration of the spin injection scheme. The bottom Pt layer invokes an injection of the pure spin current due to the spin Hall effect. (c) Reflection high energy electron diffraction images for the Pt surface (i) and the NiO surface (ii). Schematic of the crystal orientation relationship is shown in (iii). 
of the FeNi grown on top of the $\mathrm{NiO}$ under the pure spin current injection to understand the STT in the NiO layer. Unlike ferromagnetic materials, magnetic moments in oxide antiferromagnetic materials cannot be easily controlled by external magnetic field and they are tied with the crystalline magnetic anisotropy. ${ }^{16}$ In order to control the AFM magnetic moments, we develop a single crystal NiO. Nickel oxide has a rock salt structure with the lattice constant of $4.2 \AA{ }^{17}$ which is similar to the lattice constants of $\mathrm{MgO}(4.1 \AA)$ and Pt $(3.9 \AA)$ within a $5 \%$ mismatch. The magnetic moments inhabit the nickel atoms as shown in Fig. 1(a). The magnetic moments in $\{111\}$ planes are ferromagnetically coupled with its anisotropy in the $\langle-211\rangle,\langle 1-21\rangle$, and $\langle 11-2\rangle$ directions. The antiferromagnetism originates from the antiparallel coupling between the magnetic moments in the neighboring \{111\} planes by super exchange coupling. From the symmetry of these crystal planes, there are twelve possible orientations of magnetic moments, each of which makes a non-collinear angle with the [010] directions.

The layer stacks of Pt $5 \mathrm{~nm} / \mathrm{NiO} 10 \mathrm{~nm} / \mathrm{FeNi} 3 \mathrm{~nm} / \mathrm{SiO}_{2}$ $5 \mathrm{~nm}$ were prepared on $\mathrm{MgO}(001)$ single crystal substrate (Fig. 1(b)). Pt layer was formed by magnetron sputtering on $\mathrm{MgO}(001)$ with a substrate temperature of $600{ }^{\circ} \mathrm{C}$ and was then taken out to the air. After transferring the sample to another chamber for pulsed laser deposition (PLD), the sample was heated to $400{ }^{\circ} \mathrm{C}$ and its surface crystallinity was checked by reflection high energy electron diffraction (RHEED). Fig. 1(c-i) shows the RHEED pattern of the Pt surface in the direction of $\mathrm{MgO}(001)[100]$. Clear streaks indicate the atomically smooth surface of Pt layer, and the crystal orientation relative to the $\mathrm{MgO}$ is determined to be $\mathrm{MgO}(001)[001] / \mathrm{Pt}(001)[001]$. NiO layer was then deposited by PLD with an oxygen partial pressure of $2 \times 10^{-2} \mathrm{~Pa}$ at the substrate temperature of $400{ }^{\circ} \mathrm{C}$. The RHEED pattern for the surface of the $\mathrm{NiO} 10 \mathrm{~nm}$ shown in Fig. 1(c-ii) also shows clear streaks indicating the atomically smooth surface from which we determined the epitaxial orientation relationship between the $\mathrm{NiO}$ and the $\mathrm{Pt}$ layer to be $\mathrm{MgO}(001)[100] / /$ $\mathrm{Pt}(001)[100] / / \mathrm{NiO}(001)[100]$. The following $\mathrm{FeNi}$ and $\mathrm{SiO}_{2}$ layers were deposited by magnetron sputtering. The samples shown in this letter are as-deposited with no unidirectional anisotropy in FeNi. Control samples of $\mathrm{MgO}(001) / \mathrm{Pt} 5 \mathrm{~nm} /$ $\mathrm{SiO}_{2} 10 \mathrm{~nm} / \mathrm{FeNi} 3 \mathrm{~nm} / \mathrm{SiO}_{2} 5 \mathrm{~nm}$ and $\mathrm{MgO}(001) / \mathrm{Pt} 5 \mathrm{~nm} /$ FeNi $3 \mathrm{~nm} / \mathrm{SiO}_{2} 5 \mathrm{~nm}$, all made by magnetron sputtering, were grown epitaxially up to the Pt layer.

In order to investigate the spin transport in the $\mathrm{NiO}$, we performed the ST-FMR measurement, which is a useful method for quantifying the spin torque exerting in the system. The film is patterned into $4-10 \mu \mathrm{m}$ wide strips attached to a coplanar waveguide facilitating both the r.f. and d.c. current injection into the strip. The long axis of the strip along which the d.c. current flows is parallel to [100] direction of the $\mathrm{MgO}$ substrate. This ensures the spin quantization axis of the pure spin current to be in the [010] direction, thus always making a finite angle with the anisotropic magnetic moments of the epitaxially grown $\mathrm{NiO}$, and so that the spin current would exert a spin torque on the $\mathrm{NiO}$ magnetic moments. ST-FMR is performed by sweeping the external magnetic field at a fixed frequency of the r.f. current. Figure 2(a) shows the measurement configuration together with our

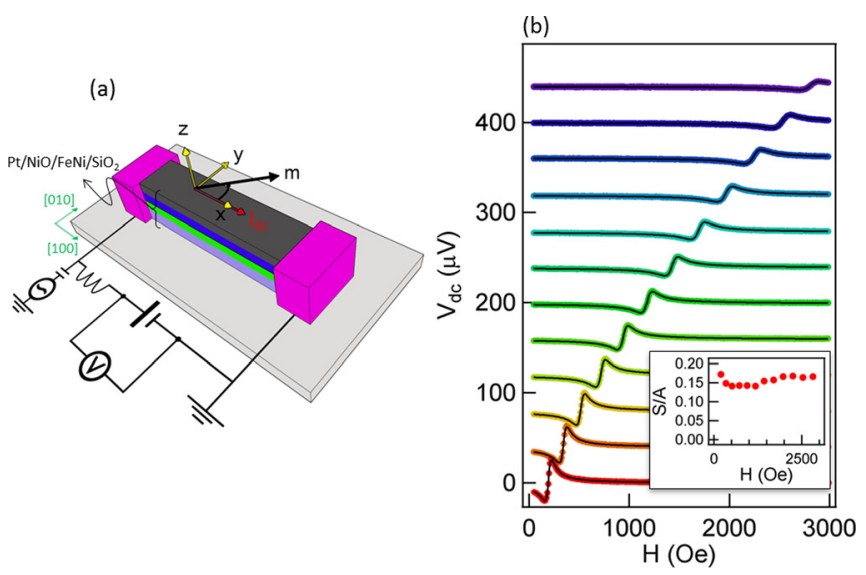

FIG. 2. (a) Measurement configuration with a ST-FMR circuitry. The strip sample is patterned so that $I_{d c}$ flows along the [100] direction of the $\mathrm{MgO}$. (b) ST-FMR spectra for Pt $5 \mathrm{~nm} / \mathrm{NiO} 10 \mathrm{~nm} / \mathrm{FeNi} 3 \mathrm{~nm} / \mathrm{SiO}_{2} 5 \mathrm{~nm}$. The inset shows the ratio of symmetric (S) and antisymmetric (A) Lorentzian magnitudes in the spectra.

coordinate system. The positive electric current is defined when it flows along the positive $x$ direction. The external positive magnetic field is applied in the sample plane and at $45^{\circ}$ away from $x$ axis. We apply nominal r.f. power up to 14 $\mathrm{dBm}$ and d.c. current up to $2.5 \mathrm{~mA}$ to the strip. All the measurements were performed at room temperature. The expected FMR spectra with the spin injection by the spin Hall effect in such a system can be composed of symmetric and antisymmetric Lorentzians. The detailed explanation can be found elsewhere. ${ }^{12,18}$ The obtained spectra shown in Fig. 2(b) for the $\mathrm{Pt} / \mathrm{NiO} / \mathrm{FeNi} / \mathrm{SiO}_{2}$ are composed of both Lorentzians and the ratio between the symmetric and antisymmetric components is found to be finite, implying that the spin torque is exerted on the FeNi layer through the $\mathrm{NiO}$. In order to further characterize the spin torque in the $\mathrm{NiO}$ layer, we mainly focused on the change in the linewidth under the spin current injection which reflects the change in effective damping due to the anti-damping spin torque due to the spin current.

Figure 3 summarizes the results obtained by the ST-FMR measurement and compares with the control samples. The most important results in this letter on the linewidth change due to the spin current are shown in Figs. 3(g)-3(i). Before discussing our central results, we would like to first show the basic FMR results obtained by the ST-FMR measurement. The ferromagnetic resonance for three different samples summarized in Figs. 3(a)-3(c) are well fitted by Kittel's formula $f=(\gamma / 2 \pi) \sqrt{\left(H_{e x}+H_{a}\right)\left(H_{e x}+H_{a}+4 \pi M_{e f f}\right)}$, where $\gamma=1.76 \times 10^{11}\left(\mathrm{~s}^{-1} \mathrm{~T}^{-1}\right)$ is gyromagnetic ratio, $H_{e x}$ is the external magnetic field, $H_{a}$ is the inplane anisotropy field, and $4 \pi M_{\text {eff }}$ is the effective demagnetizing field. The fitting yields $4 \pi M_{\text {eff }}=0.59,0.72$, and $0.6 \mathrm{~T}$ with a negligible inplane anisotropy for $\mathrm{Pt} / \mathrm{NiO} 10 \mathrm{~nm} / \mathrm{FeNi}, \mathrm{Pt} / \mathrm{FeNi}$, and $\mathrm{Pr} / \mathrm{SiO}_{2} / \mathrm{FeNi}$ samples, respectively. We separately measured the saturation magnetization $M_{S}=0.78 \mathrm{~T}$ for a single layer of the FeNi by SQUID magnetometry. The discrepancy between the saturation magnetization and the $4 \pi M_{\text {eff }}$ values may originate from the perpendicular anisotropy or magnetic dead layer at the interface. The Gilbert damping $\alpha$ of the FeNi is extracted 

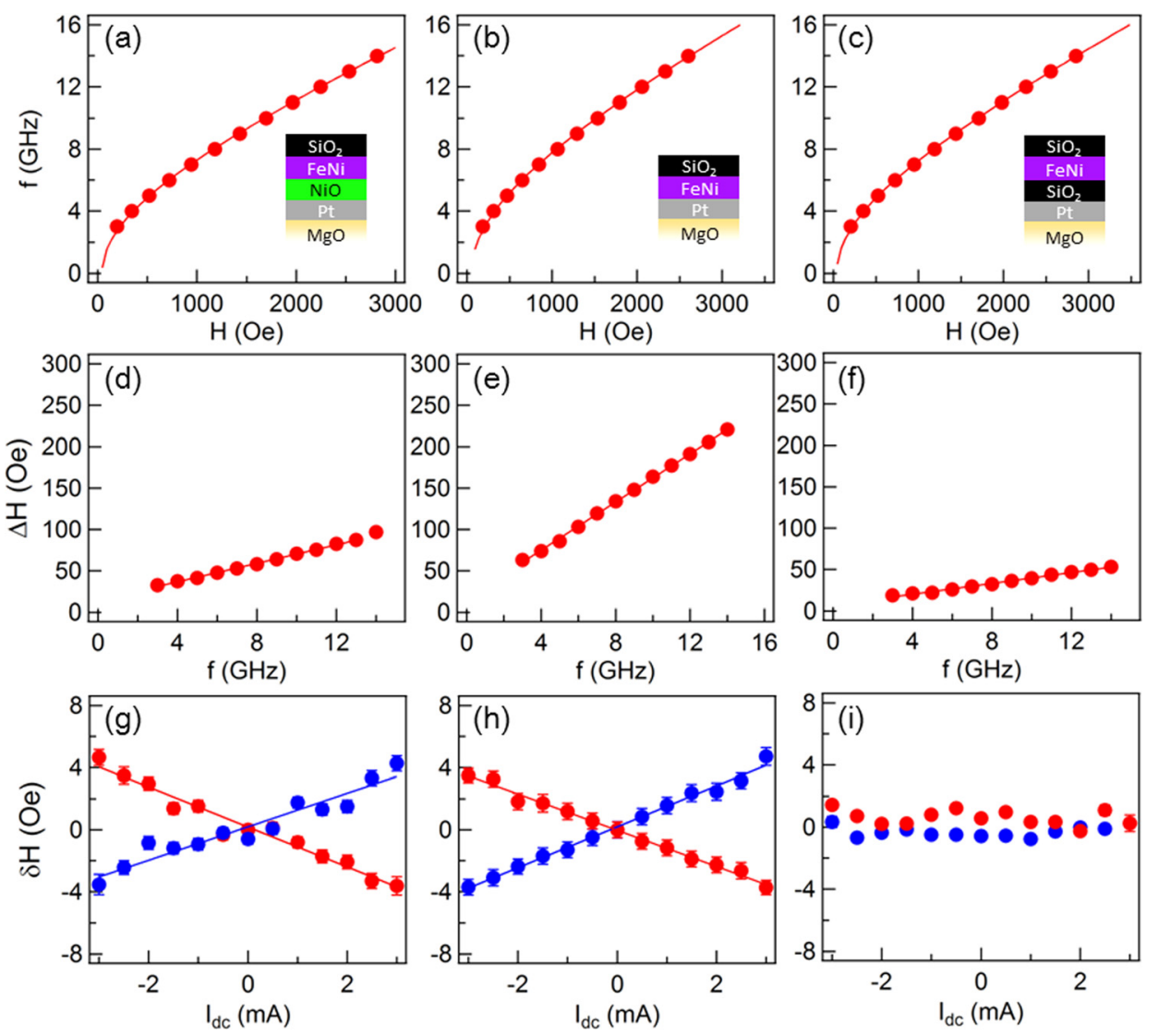

FIG. 3. Resonant frequency vs. field curves for (a) Pt $5 \mathrm{~nm} / \mathrm{NiO} 10 \mathrm{~nm} / \mathrm{FeNi}$ $3 \mathrm{~nm} / \mathrm{SiO}_{2} 5 \mathrm{~nm}$, (b) Pt $5 \mathrm{~nm} / \mathrm{FeNi}$ $3 \mathrm{~nm} / \mathrm{SiO}_{2} 5 \mathrm{~nm}$, and (c) Pt $5 \mathrm{~nm} / \mathrm{SiO}_{2}$ $10 \mathrm{~nm} / \mathrm{FeNi} 3 \mathrm{~nm} / \mathrm{SiO}_{2} 5 \mathrm{~nm}$. The dots are the experimental data and the lines are the fitting by the Kittel's formula. The FMR spectral linewidth as a function of the frequency for (d) Pt $5 \mathrm{~nm}$ / $\mathrm{NiO} 10 \mathrm{~nm} / \mathrm{FeNi} 3 \mathrm{~nm} / \mathrm{SiO}_{2} 5 \mathrm{~nm}$, (e) Pt $5 \mathrm{~nm} / \mathrm{FeNi} 3 \mathrm{~nm} / \mathrm{SiO}_{2} 5 \mathrm{~nm}$, and (f) Pt $5 \mathrm{~nm} / \mathrm{SiO}_{2} 10 \mathrm{~nm} / \mathrm{FeNi} 3 \mathrm{~nm} / \mathrm{SiO}_{2}$ $5 \mathrm{~nm}$. The change of the linewidth as a function of $I_{d c}$ at the resonance frequency of $9 \mathrm{GHz}$ for $(\mathrm{g}) \mathrm{Pt} 5 \mathrm{~nm} / \mathrm{NiO}$ $10 \mathrm{~nm} / \mathrm{FeNi} 3 \mathrm{~nm} / \mathrm{SiO}_{2} 5 \mathrm{~nm}$, (h) $\mathrm{Pt}$ $5 \mathrm{~nm} / \mathrm{FeNi} 3 \mathrm{~nm} / \mathrm{SiO}_{2} 5 \mathrm{~nm}$, and (i) $\mathrm{Pt}$ $5 \mathrm{~nm} / \mathrm{SiO}_{2} \quad 10 \mathrm{~nm} / \mathrm{FeNi} \quad 3 \mathrm{~nm} / \mathrm{SiO}_{2}$ $5 \mathrm{~nm}$. The red and the blue dots are with the positive and negative external field, respectively. The lines are the linear fitting to extract $\delta H / I_{d c}$. from the linewidth $\Delta H$ as a function of frequency by $\Delta H=\Delta H_{0}+2 \pi f \alpha / \gamma$ where $\Delta H_{0}$ is the inhomogeneous broadening. ${ }^{19}$ We obtain $\alpha=0.016,0.04$, and 0.009 and $\Delta H_{0}=14,16$, and $6 \mathrm{Oe}$ for $\mathrm{Pt} / \mathrm{NiO} 10 \mathrm{~nm} / \mathrm{FeNi}, \mathrm{Pt} / \mathrm{FeNi}$, and $\mathrm{Pr} / \mathrm{SiO}_{2} / \mathrm{FeNi}$, respectively, as the actual fittings are shown in Figs. 3(d)-3(f). Finally, the most important observations on the change of the linewidth by the spin current are shown in Figs. 3(g)-3(i). It is clearly seen that the change in the linewidth is observed in the $\mathrm{Pt} / \mathrm{NiO} / \mathrm{FeNi}$ sample as well as the $\mathrm{Pt} / \mathrm{FeNi}$ sample and not in the $\mathrm{Pt} / \mathrm{SiO}_{2} / \mathrm{FeNi}$ sample. As explained above, the d.c. current flowing in the Pt layer invokes the spin Hall effect and induces a spin accumulation at the $\mathrm{Pt} / \mathrm{NiO}$ interface. The spin torque transferred into the ferromagnetic layer should result in either increase or decrease in the linewidth as the typical $\mathrm{Pt} / \mathrm{FeNi}$ linewidth change can be seen in Fig. 3(h). With the positive spin Hall angle and the positive external field, the magnetization in FeNi feels the anti-damping torque in the positive d.c. current, leading to the decrease of the FMR linewidth. ${ }^{18}$ On the other hand, by flipping the magnetization direction with the negative external field, the positive d.c. current results in the increase of the linewidth. As the ST-FMR measurement is only sensitive to the linewidth (i.e., damping) of the FeNi layer, the change in the linewidth in $\mathrm{Pt} / \mathrm{NiO} / \mathrm{FeNi}$ can be interpreted in the way that the angular momentum is indeed transferred through the NiO. ${ }^{13}$ No linewidth change in the $\mathrm{Pt} / \mathrm{SiO}_{2} / \mathrm{FeNi}$ sample indicates that the angular momentum transport is completely impeded by non-magnetic $\mathrm{SiO}_{2}$ layer as one expects.

The relation between the linewidth and the injected spin current is given by

$$
\delta H / I_{d c}=\frac{1}{\sqrt{2}} \frac{2 \pi f}{\gamma} \frac{1}{\left(H_{e x}+2 \pi M_{e f f}\right) M_{s} t_{F M} w} \frac{\hbar}{2 e}\left(\beta \frac{\theta_{S H, P t} r_{P t}}{t_{P t}}\right),
$$

where $\delta H$ is the change in linewidth, $H_{e x}$ is the external field, $\hbar$ is the Planck's constant, $e$ is the elementary charge, $w$ is the width of the strip, $t_{F M}$ is the thickness of FeNi, $\theta_{S H, P t}$ is the spin Hall angle of the $\mathrm{Pt}, r_{P t}$ is the shunt current ratio of the $\mathrm{Pt}$ layer, and $t_{P t}$ is the thickness. Here, we introduce $\beta(0 \leq \beta \leq 1)$ as the spin-transfer efficiency in the $\mathrm{NiO}$ layer. $\beta=1$ when all of the injected spin current exerts a spin torque on the FeNi layer through the $\mathrm{NiO}$ layer (and also corresponding to the limit of zero $\mathrm{NiO}$ thickness), and $\beta=0$ when it is dissipated before reaching to the FeNi layer. Using the effective spin Hall angle $\theta_{S H, P t}$ for the $\mathrm{Pt} / \mathrm{FeNi}$ interface for both $\mathrm{Pt} / \mathrm{FeNi}$ and $\mathrm{Pt} / \mathrm{NiO}$ interfaces and the same shunt current ratios in $\mathrm{Pt}$ for both $\mathrm{Pt} / \mathrm{NiO} / \mathrm{FeNi}$ and $\mathrm{Pt} / \mathrm{FeNi}$ samples, we can simply compare $\delta H / I_{d c}$ values obtained from the slope of the plots in Figs. 3(g) and 3(h) to see how the $\mathrm{NiO}$ efficiently carries the spin angular momentum. By fitting with the data in Figs. 3(g) and 3(h), we obtained $\delta H / I_{d c}$ to be $1.1 \pm 0.1$ and $1.2 \pm 0.1 \mathrm{Oe} / \mathrm{mA}$, respectively, for $\mathrm{Pt} / \mathrm{NiO} / \mathrm{FeNi}$ and $\mathrm{Pt} / \mathrm{FeNi}$ samples. These results yield a highly efficient spin transport with $\beta=0.82$ for the $10 \mathrm{~nm} \mathrm{NiO}$ layer comparing with the previous report. $^{13}$

At finite temperatures, spin through $\mathrm{NiO}$ can be transported by the coherent Neel order dynamics as well as incoherent thermal magnons. While the incoherent contribution is expected to decay exponentially over the spin diffusion length $\lambda_{s d}$, the coherent contribution should decay 
exponentially over the so-called healing length $\lambda_{h}$ (determined by various magnetic anisotropies in $\mathrm{NiO}$ ). The experimental value $\lambda_{s}$ for the exponential decay length should be determined by $\lambda_{s} \equiv \max \left\{\lambda_{s d}, \lambda_{h}\right\}$. Both theoretical and experimental study of the temperature-dependence of the linewidth is still lacking but interesting. A study of the linewidth at lower temperatures will effectively freeze out the thermal magnons allowing one to quantify spin transport mediated purely by the coherent Neel dynamics, and the results for the linewidth as a function of $\mathrm{NiO}$ thickness at these low temperatures can be used to extract the AFM healing length.

A theory for spin transport through AFM insulators mediated by coherent Neel order dynamics (i.e., thermal contribution neglected) was developed in Refs. 7 and 20; such a theory is sufficient either at temperatures well below the Neel temperature (at which thermal magnon density is negligible) or for $\lambda_{s d} \ll \lambda_{h}$. Reference 20 studies the effects of coherent spin propagation through the AFM on the FMR linewidth in a metal|AFM|ferromagnet trilayer (akin to the current experiment) assuming an AFM with easy-axis anisotropy and the dc FMR field aligned parallel to the easy-axis. However, $\mathrm{NiO}$ is known to have a strong magnetic easyplane parallel to the (111) plane, within which a much weaker easy-axis exists, ${ }^{21}$ and the assumption of parallel alignment between the dc FMR field and the Neel easy-axis may be inapplicable to the current experiment. Further theoretical work accounting for the magnetic anisotropies and the dependences on the relative orientations between the Neel order and the dc FMR field, and further experimental characterization of the Neel vector orientation with respect to the Pt current and dc FMR field directions are clearly needed for quantitative comparisons between experiment and theory.

In summary, we performed the ST-FMR measurement on $\mathrm{NiO}$ and observed the change of the linewidth as a function of the spin current from the Pt due to the spin Hall effect. Our observation is interpreted that there is an angular momentum transfer from the Pt to the FeNi through the NiO. Our high efficient angular momentum transfer in the $\mathrm{NiO}$ comparing with the previous report on disordered $\mathrm{NiO}$ indicates that the orientation of the AFM moments is important, which presumably support the available theoretical model assuming the angular momentum transfer mediated by AFM spin fluctuations. Our results also assure that the $\mathrm{NiO}$ antiferromagnetic moments are interacting with the spin current and exciting dynamics. It would be of great interest to experimentally prove the spin torque magnetic excitation in antiferromagnet as the similar concept has already been demonstrated in ferromagnetic thin films. ${ }^{22}$

This work was partly supported by Grants-in-Aid for Scientific Research (S), Grant-in-Aid for Young Scientists (B) from Japan Society for the Promotion of Science. S.T. and Y.T. would like to acknowledge support by FAME (an SRC STARnet center sponsored by MARCO and DARPA).

${ }^{1}$ J. C. Slonczewski, J. Magn. Magn. Mater. 159, L1 (1996).

${ }^{2}$ L. Berger, Phys. Rev. B 54, 9353 (1996).

${ }^{3}$ M. Tsoi, A. G. M. Jansen, J. Bass, W. C. Chiang, M. Seck, V. Tsoi, and P. Wyder, Phys. Rev. Lett. 80, 4281 (1998).

${ }^{4}$ J. A. Katine, F. J. Albert, R. A. Buhrman, E. B. Myers, and D. C. Ralph, Phys. Rev. Lett. 84, 3149 (2000).

${ }^{5}$ A. H. MacDonald and M. Tsoi, Philos. Trans. R. Soc. A 369, 3098 (2011).

${ }^{6}$ A. C. Swaving and R. A. Duine, Phys. Rev. B 83, 054428 (2011).

${ }^{7}$ S. Takei, B. I. Halperin, A. Yacoby, and Y. Tserkovnyak, Phys. Rev. B 90, 094408 (2014).

${ }^{8}$ R. Cheng, J. Xiao, Q. Niu, and A. Brataas, Phys. Rev. Lett. 113, 057601 (2014).

${ }^{9}$ T. Kampfrath, A. Sell, G. Klatt, A. Pashkin, S. Mährlein, T. Dekorsy, M. Wolf, M. Fiebig, A. Leitenstorfer, and R. Huber, Nat. Photon. 5, 31 (2011).

${ }^{10}$ Z. Wei, A. Sharma, A. S. Nunez, P. M. Haney, R. A. Duine, J. Bass, A. H. MacDonald, and M. Tsoi, Phys. Rev. Lett. 98, 116603 (2007).

${ }^{11}$ S. Urazhdin and N. Anthony, Phys. Rev. Lett. 99, 046602 (2007).

${ }^{12}$ T. Moriyama, M. Nagata, K. Tanaka, K-J. Kim, H. Almasi, W. G. Wang, and T. Ono, e-print arXiv:1411.4100.

${ }^{13}$ H. Wang, C. Du, P. C. Hammel, and F. Yang, Phys. Rev. Lett. 113, 097202 (2014).

${ }^{14}$ J.-P. Jan, Solid State Phys. 5, 1 (1957).

${ }^{15}$ Y. Kajiwara, K. Harii, S. Takahashi, J. Ohe, K. Uchida, M. Mizuguchi, H. Umezawa, H. Kawai, K. Ando, K. Takanashi et al., Nature 464, 262 (2010).

${ }^{16}$ K. Arai, T. Okuda, A. Tanaka, M. Kotsugi, K. Fukumoto, T. Ohkochi, T. Nakamura, T. Matsushita, T. Muro, M. Oura et al., Phys. Rev. B 85, 104418 (2012).

${ }^{17}$ L. Duo, M. Finazzi, and F. Ciccacci, Magnetic Properties of Antiferromagnetic Oxide Materials: Surfaces, Interfaces, and Thin Films (Wiley-VCH Verlag GmbH \& Co. KGaA, Weinheim, 2010).

${ }^{18}$ L. Liu, T. Moriyama, D. C. Ralph, and R. A. Buhrman, Phys. Rev. Lett. 106, 036601 (2011).

${ }^{19}$ R. Arias and D. L. Mills, Phys. Rev. B 60, 7395 (1999).

${ }^{20}$ S. Takei, T. Moriyama, T. Ono, and Y. Tserkovnyak, e-print arXiv: 1502.04128

${ }^{21}$ M. Fraune, U. Ruediger, G. Guentherodt, S. Cardoso, and P. Freitas, Appl. Phys. Lett. 77, 3815 (2000).

${ }^{22}$ S. I. Kiselev, J. C. Sankey, I. N. Krivorotov, N. C. Emley, R. J. Schoelkopf, R. A. Buhrman, and D. C. Ralph, Nature 425, 380 (2003). 